\title{
Efectos del ácido giberélico, bencilaminopurina y fluridona en la germinación in vitro de Ugni molinae Turcz. (Myrtaceae)
}

\section{Effects of gibberellic acid, benzylaminopurine and fluridone on the in vitro germination of Ugni molinae Turcz. (Myrtaceae)}

\author{
Mario Rodríguez Beraud*, Nelson Hormazábal Vásquez, Ximena Araneda Durán, Jocelyne Tampe \\ Pérez, Victoria lobos Marambio \& Claudia Castillo Rubio
}

Universidad Católica de Temuco, Facultad de Recursos Naturales, Escuela de Agronomía. Rudecindo Ortega 02950, Temuco, Chile.

*marodrig@uct.cl

\begin{abstract}
RESUMEN
En la presente investigación se estudiaron los efectos de bencilaminopurina (BAP), ácido giberélico (GA $)_{3}$ y fluridona (FLU) sobre la germinación in vitro de murtilla (Ugni molinae), una especie nativa de Chile. La semilla de murtilla presenta una fuerte dormancia, razón por la cual se realizaron dos ensayos a fin de mejorar la germinación. Los resultados indican que FLU, un inhibidor de la síntesis de ABA, fue el más eficaz en romper la dormancia. La combinación de $10 \mu \mathrm{M}$ de FLU con BAP o $\mathrm{GA}_{3}$ mejoraron la germinación, siendo FLU + $\mathrm{GA}_{3}$ la más óptima, ésta fue capaz de elevar la germinación de $34 \%$ en el testigo a $96 \%$ a los 60 días. Lo anterior sugiere que en los mecanismos de dormancia de la semilla de $U$. molinae está involucrada la presencia de ABA.
\end{abstract}

Palabras clave: Dormancia, murtilla, ácido abscísico, Mirtáceas.

\begin{abstract}
In the present investigation, the effects of benzylaminopurine (BAP), gibberellic acid (GA $)$ and fluridone (FLU) on in-vitro germination of murtilla (Ugni molinae), a native species to Chile were studied. Murtilla seed has a strong dormancy, the reason why two trials were performed to improve the germination. The results show that FLU, an inhibitor of the synthesis of ABA, was the most effective in breaking dormancy. The combination of $10 \mu \mathrm{M}$ FLU with BAP or GA3 improved germination, FLU $+\mathrm{GA}_{3}$ was the optimum combination. It raised the germination from $34 \%$ in the control seed to $96 \%$ up to 60 days after treatment. The results also suggest that in the mechanisms of seed dormancy of $U$. molinae the presence of $\mathrm{ABA}$ is involved.
\end{abstract}

KeYwords: Dormancy, murtilla, abscisic acid, Myrtaceae.

\section{INTRODUCCIÓN}

Murta o murtilla (Ugni molinae Turcz.), es una Mirtácea nativa de Chile, crece de forma silvestre en el Sur de Chile, desde la Región del Maule hasta el Río Palena, está presente tanto en la Cordillera de la Costa como en la Cordillera de los Andes en los tipos forestales robleraulí-coigüe, coigüe-raulí-tepa y roble-hualo-ciprés de las Guaitecas (Hoffmann 2005). Tanto hojas como frutos poseen numerosas características nutritivas y medicinales como efectos analgésicos, anti-inflamatorios, astringentes, actividad antimicrobiana y antioxidante (Hauser et al. 2014, Suwalsky \& Avello 2014).
La germinación in vitro se utiliza con éxito para muchas especies y ha demostrado ser superior a otras técnicas como la germinación ex vitro en sustratos o con papel filtro (Ghanbari et al. 2012). La germinación in vitro permite producir plántulas asépticas como fuente de explantes (Larson et al. 2006), la producción de híbridos interespecíficos (Pereira-Netto et al. 2006), la producción de híbridos somáticos (Correia \& Canhoto 2010), la producción de callos (Rodríguez et al. 2014a), la inducción a la poliploidía (Dhooghe et al. 2011), y la germinación de semillas provenientes de cruzamientos controlados para programas de mejoramiento vegetal, donde la semilla es un material muy valioso y escaso (Rodríguez 2011). En las 
especies nativas es frecuente observar una fuerte latencia o dormancia en sus semillas, lo que les permite sobrevivir y eludir las condiciones ambientales desfavorables, en cambio en especies cultivadas los mecanismos de latencia han sido atenuados por efecto de la selección artificial y domesticación, facilitando con ello su manejo productivo (Bewley et al. 2013).

En condiciones naturales, la semilla de murtilla tiene un sistema de dispersión endozoocoria, dado por la ingestión de los frutos por algunos animales (Figueroa 2003), presentando una baja germinación respecto a otras Mirtáceas, producto de una fuerte dormancia (Smith-Ramírez et al. 1998, Rodríguez et al. 2014b). La dormancia o latencia en semillas se manifiesta de distintas formas según la especie, consiste en la interrupción de los acontecimientos internos de una semilla viable para completar su germinación bajo condiciones propicias. Baskin \& Baskin (2004) definen como semilla en dormancia, aquella que no tiene la capacidad de germinar durante un período específico de tiempo bajo una combinación de factores físicos y medioambientales que de otro modo serían favorables para la germinación. En la dormancia endógena o embrionaria el embrión impide la germinación, la que puede ser causada por un mecanismo de inhibición fisiológica del embrión (inhibición metabólica, incremento del ácido abscísico y producción de compuestos fenólicos) o relacionada con la inhibición de la germinación durante la etapa de desarrollo y crecimiento del embrión (morfológica) (Finch-Savage \& Leubner-Metzger 2006).

El control de la dormancia ha sido atribuido a varios reguladores inhibidores, tales como ácido abscísico (ABA), y promotores como las giberelinas (GAs), citoquininas y etileno (Linkies \& Leubner-Metzger 2012, Wilson et al. 2014a). El ácido abscísico (ABA) es un sesquiterpeno obtenido a partir de carotenoides, y está involucrado en la dormancia de semillas para muchas especies. Se inactiva irreversiblemente por hidroxilación, dando lugar al ácido faseico y dihidrofaseico, o reversiblemente por conjugación con monosacáridos (Baskin \& Baskin 2014). Se ha relacionado la dormancia de ABA con la inducción de la síntesis de proteínas de almacenamiento en las semillas e inhibición de la síntesis de proteínas relacionadas con la movilización de reservas (Kermode 2005). Durante el desarrollo de la semilla, el contenido de ABA inicial es bajo, aumentando rápidamente para alcanzar un máximo a la mitad o finales de la maduración, quedando así la dormancia primaria establecida y luego declina gradualmente durante la desecación y almacenamiento de la semilla (Gubler et al. 2005).

Existen inhibidores del ABA como, fluridona (FLU), un producto de origen orgánico, inhibidor de la enzima fitoeno desaturasa, que convierte el fitoeno a fitoflueno en la vía de la biosíntesis de carotenoides (Kondhare et al. 2014). Los carotenoides son los principales precursores del ABA en plantas, por lo tanto, la inhibición de la carotenogénesis también implica impedir la biosíntesis de ABA. Muchos investigadores han encontrado en inhibidores de ABA, como fluridona y norfluorazon, comúnmente utilizados como herbicidas (Sun et al. 2012), una herramienta útil para estudios de germinación y rompimiento de la dormancia tanto para embriones cigóticos (Popova 1995, Koornneef et al. 2002) como somáticos (Garcês et al. 2014). El balance entre ABA y niveles de GAs tiene un rol importante en la regulación de la dormancia. El ABA induce la promoción y mantención de la dormancia, mientras que las GAs especialmente $\mathrm{GA}_{3}$, promueve la progresión para el inicio de la germinación. Las señales del medio ambiente regulan este balance modificando la expresión de enzimas biosintéticas y catabólicas (Finkelstein et al. 2008). La regulación del nivel de dormición es consecuencia de un balance dinámico entre los procesos de síntesis y degradación del ABA y de las GAs, donde la salida de la dormición se asocia con un incremento en la concentración de GAs (Sawada et al. 2008), cambios en los tejidos a la sensibilidad a las GAs (Benech-Arnold et al. 2006), una reducción del contenido de ABA (Millar et al. 2006) y una pérdida en la sensibilidad a este compuesto (Gualano et al. 2007).

Una manera eficiente de estudiar los efectos de productos químicos que promueven la germinación es la adición de ellos a medios de cultivo para la germinación in vitro, en relación a esto, la hipótesis planteada es que es posible romper latencia de la semilla de $U$. molinae a través de tratamientos de giberelina $\left(\mathrm{GA}_{3}\right)$, bencilaminopurina (BAP) y fluridona (FLU). Los objetivos de esta investigación son determinar las concentraciones adecuadas de $\mathrm{GA}_{3}$, BAP y FLU, como también estudiar los efectos combinados de estos productos sobre la germinación de murtilla, para poder recomendar un tratamiento químico eficaz para romper la fuerte dormancia que presentan las semillas de esta especie.

\section{MATERIALES Y MÉTODOS}

Se realizaron dos ensayos. El primero, para determinar la mejor concentración $(1,10$ y $100 \mu \mathrm{M})$ de $\mathrm{GA}_{3}$, BAP y FLU más un testigo, totalizando 10 tratamientos y el segundo para ver los potenciales efectos combinados entre los mejores tratamientos de $\mathrm{GA}_{3}$ y $\mathrm{BAP}$ con el mejor tratamiento de FLU, totalizando seis tratamientos $\left(\mathrm{GA}_{3} 10 \mu \mathrm{M}\right.$, BAP 10 $\mu \mathrm{M}$, FLU $10 \mu \mathrm{M}, \mathrm{GA}_{3} 10 \mu \mathrm{M}+\mathrm{FLU} 10 \mu \mathrm{M}$, BAP $10 \mu \mathrm{M}+$ FLU $10 \mu \mathrm{M}$, y testigo).

\section{Material VEGETAL}

Las semillas utilizadas en los ensayos de germinación se obtuvieron de frutos cosechados el 2009 de una población de murtillas de la localidad de Curiñanco, sector costero de Valdivia, Chile $39^{\circ} 40^{\prime} \mathrm{S}, 73^{\circ} 21^{\prime} \mathrm{O}$. Los frutos maduros fueron colocados en maceración por 5 días, con un cambio de agua diario, al final se separó la semilla del resto de la 
pulpa y se secaron en cámara a $25 \pm 2{ }^{\circ} \mathrm{C}$. Las semillas secas fueron almacenadas por seis meses a $4{ }^{\circ} \mathrm{C}$.

DESINFECCIÓN DE SEMILLA

Las semillas utilizadas para el ensayo de germinación fueron desinfectadas en cámara de flujo laminar con tres soluciones en forma secuencial (fungicidas, alcohol y cloro). La solución de fungicidas $\left(2 \mathrm{~g} \mathrm{~L}^{-1}\right.$ de Mancozeb, 0,6 $\mathrm{g} \mathrm{L}^{-1}$ de Benomil, más unas gotas de Tween 20) se aplicó a las semillas bajo agitación constante por $20 \mathrm{~min}$, luego se les adicionó etanol $70^{\circ}$ por $5 \mathrm{~s}$ e hipoclorito de sodio $(\mathrm{NaOCl})$ al $2 \% \mathrm{v} / \mathrm{v}$ (cloro activo) por $10 \mathrm{~min}$, finalizando con tres enjuagues consecutivos con agua destilada estéril.

\section{Prueba de Viabilidad de Semillas}

Se determinó la viabilidad de las semillas desinfectadas a través del test de tetrazolio en cinco repeticiones de 25 semillas cada una (ISTA 2001). A las semillas se les realizó un pequeño corte de la testa en la zona micropilar y se le aplicó una solución acuosa al 1\% de cloruro de 2, 3, 5 trifenil tetrazolio reposando en esta solución por 24 h a 30 ${ }^{\circ} \mathrm{C}$ en oscuridad. Se consideró viable aquella semilla que tenía el embrión completamente teñido rojo.

\section{PREPARACIÓN DE MEDIOS DE CULTIVO}

Los medios de cultivo consistieron en agua destilada gelificada con $7 \mathrm{~g} \mathrm{~L}^{-1}$ de agar microbiológico ${ }^{\circledR}$ Merck, ajustando el pH a 5,8 con 1,0 N hidróxido de potasio $(\mathrm{KOH})$ y $1,0 \mathrm{~N}$ de ácido clorhídrico $(\mathrm{HCl})$. Los medios fueron esterilizados en autoclave por $20 \mathrm{~min}$ a $121^{\circ} \mathrm{C}$ a $1 \mathrm{~atm}$ de presión, para luego dosificar en cámara de flujo laminar 10 $\mathrm{mL}$ de medio por placa Petri desechable estéril de $60 \times 15$ $\mathrm{mm}$.

\section{SiEMBRA Y CONDICIONES DE CULTIVO}

Las semillas desinfectadas fueron sembradas en condiciones estériles bajo una cámara de flujo laminar. Se sembraron un total de 25 semillas por placa con un marco de siembra de $5 \mathrm{x}$ 5 unidades. Al finalizar la siembra las placas fueron selladas con parafilm $\mathrm{M}^{\circledR}$ y luego incubadas en cámara de cultivo a $25 \pm 1{ }^{\circ} \mathrm{C}$, con tubos fluorescentes de luz fría blanca en un fotoperiodo de $16 \mathrm{~h} \mathrm{luz}, 8 \mathrm{~h}$ de oscuridad y una intensidad lumínica de $50 \mu \mathrm{mol} \mathrm{m}^{-2} \mathrm{~s}^{-1}$ (tubos Philips TLD 36W/54).

\section{AnÁLISIS ESTADísticos}

Se utilizó un diseño completamente al azar para todos los tratamientos. Los gráficos fueron realizados con el programa SigmaPlot 10.0. La unidad experimental consistió en una placa con 25 semillas cada una. Cada tratamiento constó de cuatro placas. Se evaluó el porcentaje de germinación diariamente hasta los 60 días de la siembra. Los resultados se expresan como promedio \pm el error estándar. Para el caso de germinación máxima $\left(\mathrm{G}_{\max }\right)$ se realizó un análisis de varianza (ANDEVA) y la prueba de Duncan con el programa SPSS versión 15.0 para lo cual los porcentajes de germinación se transformaron al arcoseno de la raíz cuadrada del porcentaje dividido por 100 .
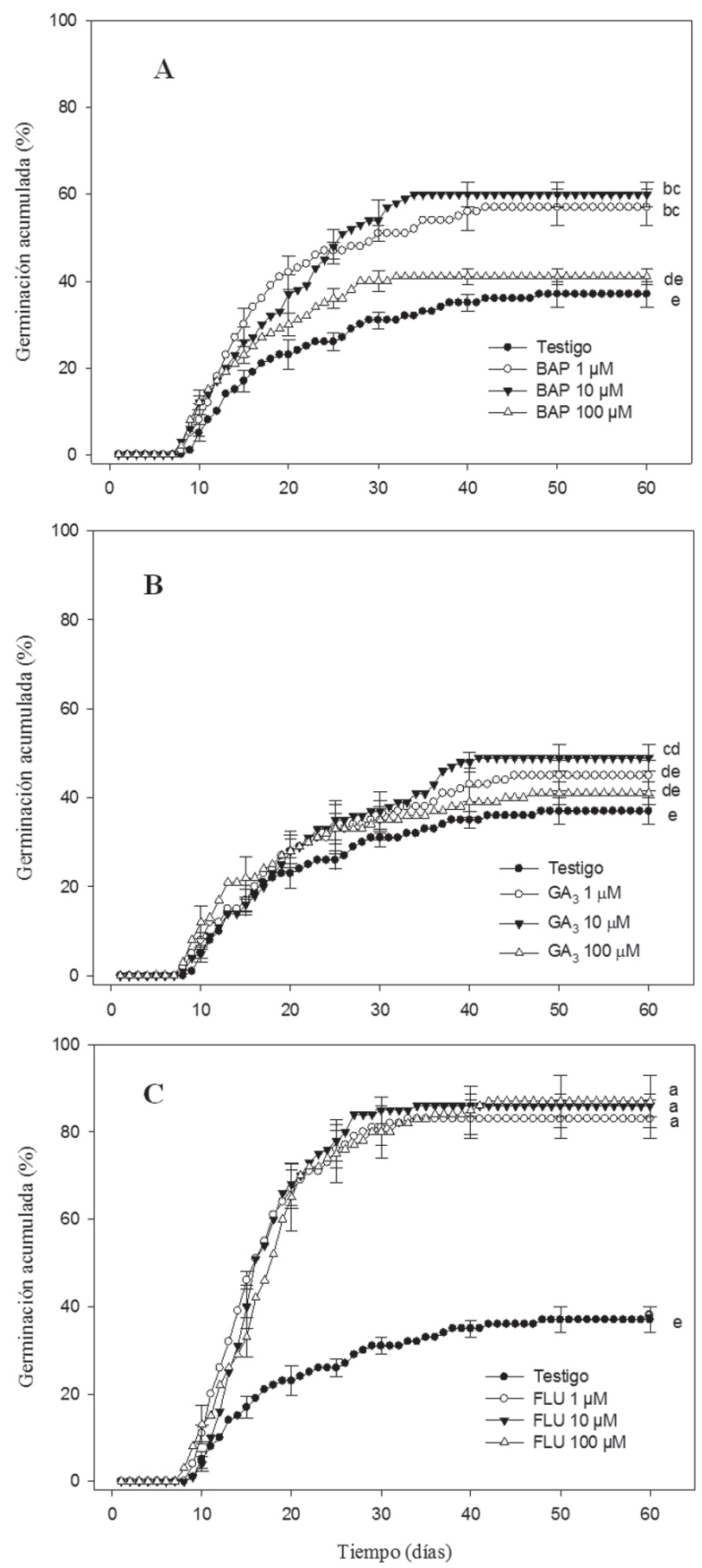

Figura 1. Porcentaje de germinación acumulada de $U$. molinae bajo diferentes concentraciones de BAP, GA $\mathrm{G}_{3}$ y FLU. Barras verticales representan el error estándar del promedio. Letras distintas son diferentes significativamente, de acuerdo al test de Duncan $(\mathrm{p} \leq 0,05)$.

FIgURE 1. Cumulative germination percentages of $U$. molinae under different concentrations of BAP, GA and FLU. Vertical bars represent the standard error of mean. Different letters are significantly different according to Duncan test $(\mathrm{p} \leq 0.05)$. 


\section{RESULTADOS}

La prueba de tetrazolio determinó un 97\% de viabilidad para las semillas desinfectadas. Las semillas viables presentaron embriones de color rojo intenso con una zona central no teñida, correspondiente al eleosoma.

ENSAyo 1. EFECTO de LA CONCENTRACión de GA 3 , BAP y FLU EN LA GERMINACIÓN IN VITRO DE U. MOLINAE

Independiente de los tratamientos utilizados, la semilla de murta inició su germinación a partir del noveno día (Fig. 1A, 1B y 1C). En general, los productos químicos estimularon la germinación respecto al testigo que alcanzó una germinación máxima $\left(\mathrm{G}_{\max }\right)$ de $37 \%$. En los tratamientos con BAP las concentraciones de 1 y $10 \mu \mathrm{M}$ lograron diferencias significativas respecto al testigo (57 y $60 \%$ respectivamente) en cambio $\mathrm{GA}_{3}$ solo fue superior con $10 \mu \mathrm{M}$ (49\%). El incremento de la concentración de los químicos aplicados de 10 a $100 \mu \mathrm{M}$ en BAP y GA $\mathrm{A}_{3}$ no logró elevar significativamente la germinación. Los tratamientos con FLU fueron los que obtuvieron los mayores valores promedios (mayor a 80\%) (Fig. 2), siendo eficaz en todas las concentraciones empleadas y significativamente superior al testigo (Fig. 1C). No se observó un efecto depresor a concentraciones altas $(100 \mu \mathrm{M})$ como fue el caso de BAP y GA . $_{3}$.

Por otra parte, el tiempo medio para alcanzar la germinación máxima (TM, momento en que se alcanza el $50 \%$ del valor $\mathrm{G}_{\max }$ ) osciló entre 13 a 19 días en los tratamientos de $\mathrm{GA}_{3}$ con $100 \mu \mathrm{M}$ y $10 \mu \mathrm{M}$ respectivamente, encontrándose los demás tratamientos entre estos rangos.

El patrón sigmoidal de las curvas de germinación acumulada (Fig. 1A, 1B y 1C) está precedido de una fase comprendida entre la siembra y el inicio de germinación (0-8 días), luego se inicia una fase exponencial donde los porcentajes de germinación ocurren en forma acelerada para luego empezar a desacelerarse y finalmente quedar en forma estacionaria con poco o ningún incremento respecto a la germinación, esta última fase se inicia después de los 30 días en la semilla tratada con FLU y después de los 40 días para el caso de los ensayos con $\mathrm{GA}_{3}$ y BAP.

Ensayo 2. Estudio de LA INTERACCiÓn de GA 3 Y BAP CON FLU SOBRE LA GERMINACIÓN IN VITRO DE U. MOLINAE

Todos los tratamientos químicos fueron significativamente superiores al testigo $(\mathrm{p} \leq 0,05)$. Los tratamientos de $\mathrm{GA}_{3}$, BAP y FLU en concentración de $10 \mu \mathrm{M}$ favorecieron la germinación (72, 68 y 81\%, respectivamente) en relación al testigo, el que obtuvo un 39\% a los 60 días (Fig. 3), como lo observado en el ensayo anterior. La combinación de FLU con $\mathrm{GA}_{3}$ o BAP logró un efecto sinérgico importante, superando los altos porcentajes de FLU por sí solo, alcanzando 96 y 92\% a los 60 días respectivamente, que son los valores más altos registrados para esta investigación, haciendo germinar casi la totalidad de la semilla viable. Al analizar la cinética se puede observar que los tratamientos combinados aunque no inciden en el inicio de la germinación, consiguen acelerar la curva de germinación logrando en 20 días posterior a la siembra, la germinación de más del $85 \%$ de la semilla, con un TM igual a 13 días en ambos casos, convirtiendo a estos tratamientos en los más promisorios para germinar semilla de murtilla.

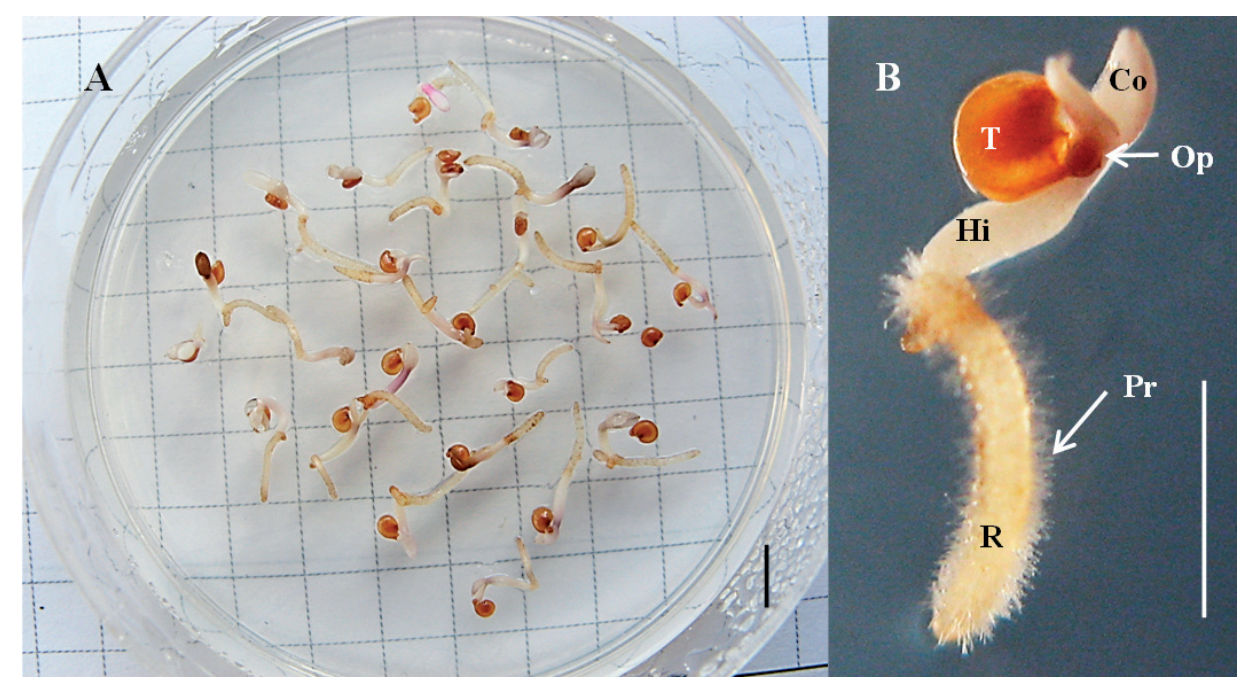

Figura 2. Germinación de semilla de U. molinae en presencia de $10 \mu \mathrm{M}$ de Fluridona. A) Germinación in vitro en placa Petri. B) Detalle de semilla germinada. Cotiledón (Co), testa (T), opérculo (Op), hipocótilo (Hi), pelos radicales (Pr) y raíz (R). Barras: 5 mm.

FIGURE 2. Seed germination U. molinae in presence of $10 \mu \mathrm{M}$ Fluridone. A) In vitro germination in Petri dish. B) Detail of germinated seed. Cotyledon $(\mathrm{Co})$, testa $(\mathrm{T})$, cover $(\mathrm{Op})$, hypocotyl $(\mathrm{Hi})$, root hairs $(\mathrm{Pr})$ and root $(\mathrm{R})$. Bars: $5 \mathrm{~mm}$. 


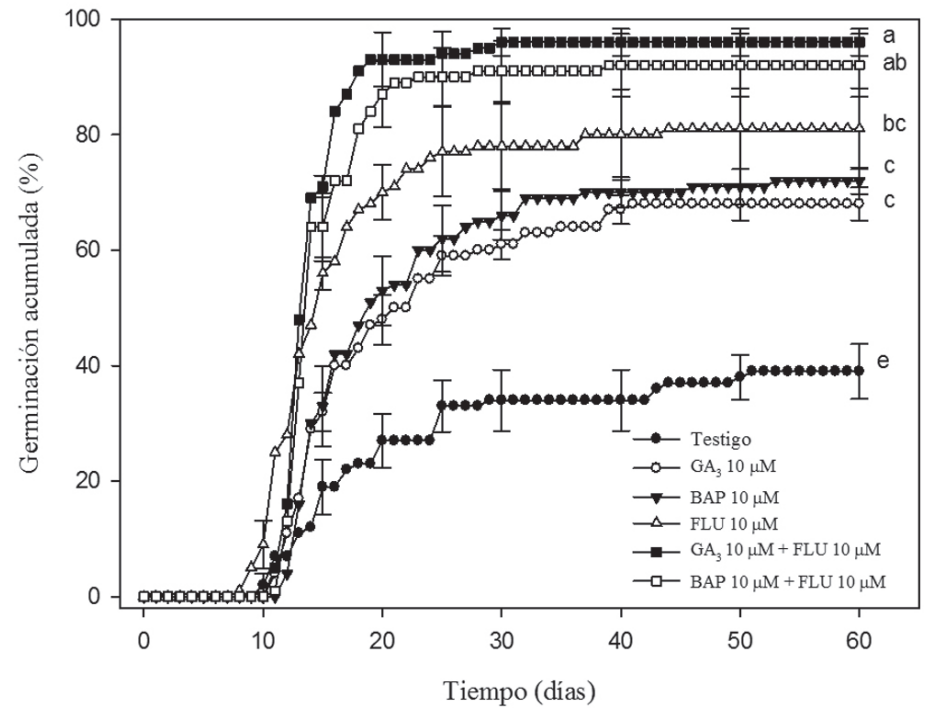

FiguRA 3. Porcentaje de germinación acumulada de U. molinae bajo efectos simples o combinados de FLU con BAP y GA. Letras distintas son significativamente diferentes de acuerdo al test de Duncan $(\mathrm{p} \leq 0,05)$.

FigURE 3. Cumulative germination percentages of $U$. molinae under simples or combined effects of FLU with BAP and GA. Differents letters are significantly different according to Duncan test $(\mathrm{p} \leq 0.05)$.

\section{DISCUSIÓN}

La elevada viabilidad y baja germinación de los tratamientos testigos corroboran la tesis que la semilla de murtilla presenta una fuerte dormancia. Tanto $\mathrm{GA}_{3}$, BAP y FLU en concentración de $10 \mu \mathrm{M}$ fueron capaces de romper la dormancia de una buena proporción de la semilla tratada.

Si bien a BAP no se le atribuye un rol protagónico en la germinación como a la interacción AIB-GAs (Linkies \& Leubner-Metzger 2012), algunos investigadores como Hossain et al. (2010), Moradi \& Otroshy (2012) han mejorado la germinación con el uso de BAP, como las encontradas en el presente trabajo. Las citoquininas están presentes en el desarrollo de la semilla y se acumulan preferentemente en el endospermo e intervienen en las actividades fisiológicas y metabólicas de las semillas. El BAP activa la división celular en el embrión contribuyendo a su desarrollo (Kucera et al. 2005). Las citoquininas aunque no están involucradas directamente en el quiebre de la dormancia, permiten disminuir el nivel de inhibidores de la germinación especialmente de ABA y hacen a la semilla más sensible a las GAs (Bewley et al. 2013, Guan et al. 2014).

Por otra parte, para muchos autores el $\mathrm{GA}_{3}$ ha sido el tratamiento más efectivo para romper la dormancia (Khanna et al. 2013, Baskin \& Baskin 2014). Al respecto, dos mecanismos de acción se han propuesto para explicar el papel de las GAs en el control de la germinación. Uno es la inducción de la expresión de los genes que codifican las enzimas que hidrolizan en el endospermo sustancias de reserva a moléculas más sencillas, para que el embrión disponga de moléculas estructurales y de la energía necesaria para iniciar la síntesis de sus propias moléculas (Rayorath et al. 2008). Un segundo mecanismo consiste en un estímulo directo sobre el potencial de crecimiento del embrión, según lo sugerido para Arabidopsis thaliana (L.) Heynh. (Karssen \& Lacka 1986). No obstante, este crecimiento está condicionado ya que puede ser restringido por el ácido abscísico producido en el embrión, generándose entre $\mathrm{ABA}$ y $\mathrm{GA}_{3}$ una relación antagónica respecto a la germinación (Karssen et al. 1983, Kim et al. 2014). Piskurewicz et al. (2009) encontraron que en $A$. thaliana las GAs promueven la ruptura de la testa, opuestamente ABA reprime principalmente la ruptura del endospermo con independencia de los niveles de GAs. Araya et al. (2000) y Pedroza-Manrique \& Tupaz-Villacorte (2008) en sus investigaciones, han obtenido efectos similares a los de este ensayo en las especies Alnus acuminata Kunth e Ilex kunthiana Triana \& Plachon, respectivamente. En algunos casos, la aplicación exógena de GAs puede sustituir la estratificación en frío, como en Michelia yunnanensis Franch (Han et al. 2010), Myrica rubra A.Chev. (Chen et al. 2008) o como en Annona muricata L. que puede tener efectos sinérgicos al combinarlo con estratificación (Lobo et al. 2007), o con escarificación como en Cyclocarya paliurus (Batal) Iljinskaja (Fang et al. 2006).

Otras hormonas también tienen un rol promotor en la germinación, como los brasinoesteroides, que incrementan la biosíntesis de etileno y estimulan la elongación del hipocótilo en presencia de luz (Steber \& McCourt 2001, 
Piotrowska \& Bajguz 2011). Contrariamente, los jasmonatos la inhiben (Linkies \& Leubner-Metzger 2012, Song et al. 2014).

Al analizar la Fig. 3 queda claro que $\mathrm{GA}_{3}$ y BAP estimulan la germinación cuando se combinan con FLU, un inhibidor de la síntesis de ABA, dicho de otra forma, el rompimiento de la dormancia de la semilla de murtilla es mayor cuando los niveles de ABA son reducidos en presencia de $\mathrm{GA}_{3}$ o BAP exógeno.

En la biosíntesis de giberelinas en $A$. thaliana se han estudiado algunos genes que codifican para enzimas que activan o inactivan giberelinas, por ejemplo la enzima $\mathrm{GA}_{3}-$ oxidasa cataliza la conversión de formas inactivas a formas activas ( $\mathrm{GA}_{9}$ a $\mathrm{GA}_{4}$, o $\mathrm{GA}_{20}$ a $\left.\mathrm{GA}_{1}\right)$, la enzima $\mathrm{GA}_{2}$-oxidasa convierte formas activas en inactivas $\left(\mathrm{GA}_{4}\right.$ a $\mathrm{GA}_{34}$, o GA $\mathrm{a}$ $\mathrm{GA}_{8}$ ) (Nonogaki et al. 2010).

En condiciones favorables para la germinación, la semilla embebida incrementa los niveles de GAs. En paralelo, los niveles de ABA descienden rápidamente (Ogawa et al. 2003). Durante el desarrollo de la semilla, los contenidos de ABA son inicialmente bajos, aumentando rápidamente hasta alcanzar un punto máximo alrededor de la mitad de la etapa de maduración, después de este punto su función es limitada porque su concentración declina gradualmente durante la desecación de la semilla hasta llegar por debajo de un nivel de inhibición (Gubler et al. 2005). Las GAs se requieren para superar este estado inactivo ABA-inducido (Bewley et al. 2013). Es importante el nivel real del ABA durante la imbibición; podemos encontrar que los niveles de ABA aumentan durante la imbibición en semillas dormantes y no en las semillas no-dormantes (Debeaujon \& Koornneef 2000, Grappin et al. 2000).

La aplicación de FLU estimuló sustancialmente la germinación como lo observado en diversas especies tales como Crithmum maritimum L. (Atia et al. 2009), A. thaliana (Je et al. 2014), y Panicum virgatum L. (Duclos et al. 2014). La alta germinación con FLU sugiere que el ABA está involucrado con la dormancia de $U$. molinae, al igual que en muchas especies (Kusumoto et al. 2006, Feurtado et al. 2007). La biosíntesis de ABA puede ser bloqueada con el uso de FLU que interfiere en la biogénesis de carotenos, por tanto bloquea la acumulación de éstos y por consiguiente las plántulas germinadas poseen una apariencia albina como se observa en la Fig. 2A y 2B, debido a la oxidación de la clorofila en ausencia de carotenoides (Chen et al. 2008, Goggin et al. 2010). Se postula que el ABA está involucrado en la dormancia de la semilla de U. molinae ante situaciones de estrés salino (Rodríguez et al. 2014a), como también ocurre con A. thaliana (Wilson et al. 2014b). Otros investigadores han logrado el rompimiento de la dormancia utilizando norflurazon, otro inhibidor de la biosíntesis de carotenoides (Ali-Rachedi et al. 2004, Kusumoto et al. 2006).

Por otra parte, la sinergia observada en el caso de FLU y $\mathrm{GA}_{3}$ puede ser explicada, porque $\mathrm{ABA}$ y $\mathrm{GA}_{3}$ tienen roles protagónicos y antagónicos en la germinación. ABA establece la dormancia durante la maduración del embrión, mientras que $\mathrm{GA}_{3}$ es necesaria para romperla, de esta forma contenidos bajos de ABA promueven la biosíntesis de giberelinas, mientras que contenidos altos regulan su desactivación (Steber \& McCourt 2001, Nonogaki et al. 2010).

\section{CONCLUSIONES}

La semilla de $U$. molinae posee una fuerte dormancia, la cual puede romperse a través de la aplicación de BAP, GA y FLU especialmente en concentraciones de 1 y $10 \mu \mathrm{M}$. De los tratamientos aplicados, el FLU fue el más efectivo, lo que sugiere que en la dormancia de murtilla está involucrado el ABA. Por otra parte, el bloqueo en la producción de ABA provocado por FLU sumado a la adición de $\mathrm{GA}_{3} \mathrm{y}$ BAP produjeron un efecto sinérgico, resultando en una germinación superior al 90\%. De esta manera, se ha generado un protocolo eficiente para promover la germinación a fin de aprovechar al máximo la semilla, ya sea con fines de propagación o mejoramiento vegetal.

\section{BIBLIOGRAFÍA}

Ali-Rachedi, S., D. Bouinot, M. Wagner, M. Bonnet, B. Sotta, P. Grappin \& M. Jullien. 2004. Changes in endogenous abscisic acid levels during dormancy release and maintenance of mature seeds: studies with the Cape Verde Islands ecotype, the dormant model of Arabidopsis thaliana. Planta 219: 479-488.

Araya, E., L. Gómez, N. Hidalgo \& R. Valverde. 2000. Efecto de la luz y ácido giberélico sobre la germinación in vitro de Alnus acuminata. Agronomía Costarricense 24: 75-80.

Atia, A., A. Debez, Z. Barhoumi, A. Smaoui \& Ch. Abdelly. 2009. $\mathrm{ABA}, \mathrm{GA}_{3}$, and nitrate may control seed germination of Crithmum maritimum (Apiaceae) under saline conditions. Comptes Rendus Biologies 332: 704-710.

BASKIN, C. \& J. BASKIN. 2014. Seeds: ecology, biogeography, and evolution of dormancy and germination. Second edition. Elsevier, San Diego, United State of America. 1573 pp.

BASKIN, J.M. \& C.C. BASKIN. 2004. A classification system for seed dormancy. Seed Science Research 14: 1-16.

Benech-Arnold, R.L., N. Gualano, J. Leymarie, D. Côme, \& F. Corbineau. 2006. Hipoxia interferes with ABA metabolism and increases ABA sensitivity in embryos of dormant barley grains. Journal of Experimental Botany 57: 1423-1430.

Bewley, J.D., K. Bradford, H. Hilhorst \& H. Nonogaki. 2013. Seeds: physiology of development, germination and dormancy. 3rd ed. Springer. New York. 392 pp.

Chen, H., J. Zhang, M. Neff, S. Hong, H. Zhang, X. Deng \& L. XIONG. 2008. Integration of light and abscisic acid signaling during seed germination and early seedling 
development. Proceedings of the National Academy of Sciences 105(11): 4495-4500.

Correia, S. \& J. CAnhoto. 2010. Characterization of somatic embryo attached structures in Feijoa sellowiana Berg. (Myrtaceae) Protoplasma 242: 95-107.

Debeaujon, I. \& M. Koornneef. 2000. Gibberellin requirement for Arabidopsis seed germination is determined both by testa characteristics and embryonic abscisic acid. Plant Physiology 122: 415-424.

Dhooghe, E., K. Van Laere, T. Eeckhaut, L. Leus \& J. Van Huylenbroeck. 2011. Mitotic chromosome doubling of plant tissues in vitro. Plant Cell Tissue and Organ Culture 104: 359-373.

Duclos, D., C. Altobello \& A.G. Taylor. 2014. Investigating seed dormancy in switchgrass (Panicum virgatum L.): elucidating the effect of temperature regimes and plant hormones on embryodormancy. Industrial Crops and Products 58: 148-159.

FAng, S., J. WANG, Z. Weiet \& Z. Zhu. 2006. Methods to break seed dormancy in Cyclocarya paliurus (Batal) Iljinskaja. Scientia Horticulturae 110: 305-309.

Feurtado, J., J. Yang, S. Ambrose, A. Cutler, S. Abrams \& A. KERMODE. 2007. Disrupting abscisic acid homeostasis in western white pine (Pinus monticola Dougl. ex D. Don) seeds induces dormancy termination and changes in abscisic acid catabolites. Journal of Plant Growth Regulation 26: 46-54.

FigueroA J. 2003. Seed germination in temperate rain forest species of southern Chile: chilling and gap dependency germination. Plant Ecology 166: 227-240.

Finch-Savage, W.E. \& G. Leubner-Metzger. 2006. Seed dormancy and the control of germination. New Phytologist 171(3): 501-523.

Finkelstein, R., W. Reeves, T. Ariizumiet \& C. Steber. 2008. Molecular aspects of seed dormancy. Annual Review of Plant Biology 59: 387-415.

Garcês, H., D. Koenig, B. Townsley, M. Kim \& N. Sinha. 2014. Truncation of leafy cotyledon protein is required for asexual reproduction in Kalanchoë daigremontiana. Plant Physiology 165: 196-206.

Ghanbari, T., B. Hosseini \& Z. JabBarZadeh. 2012. Improving Salvia sclarea $\mathrm{L}$. seed germination under in vitro condition. International Journal of Agriculture: Research and Review 2(S): 1051-1058.

Goggin, D.E., R.J. Neil, S.B. Powlesa \& K. J. Steadmanc. 2010. Initial characterisation of low and high seed dormancy populations of Lolium rigidum produced by repeated selection. Journal of Plant Physiology 167: 1282-1288.

Grappin, P., D. Bouinot. B. Sotta, E. Miginiac \& M. Jullien. 2000. Control of seed dormancy in Nicotiana plumbaginifolia: Post-imbibition abscisic acid synthesis imposes dormancy maintenance. Planta 210: 279-285.

Gualano, N., F. Carrari, M.V. Rodríguez, L. Pérez-Flores, R.A. Sánchez, N. Iusem \& R. L. Benech-Arnold. 2007. Reduced embryo sensitivity to abscisic acid in a sprouting susceptible Sorghum (Sorghum bicolor) variety is associated with altered ABA signalling. Seed Science Research 17: 81-90

Guan, Ch., X. Wang, J. Feng, S. Hong, Y. Liang, B. Ren \& J. Zuo. 2014. Cytokinin antagonizes abscisic acid- mediated inhibition of cotyledon greening by promoting the degradation of abscisic acid insensitive protein in Arabidopsis. Plant Physiology 164: 1515-1526.

Gubler, F., A. Millar \& J. Jacobsen. 2005. Dormancy release, $\mathrm{ABA}$ and pre harvest sprouting. Current Opinion in Plant Biology 8: 183-187.

Han, C., G. Welbaumc \& C. Long. 2010. Seed dormancy and germination of Michelia yunnanensis (Magnoliaceae). Scientia Horticulturae 124: 83-87.

Hauser, C., A. Peñaloza, F. Rodríguez, A. Guarda \& M.J. Galotto. 2014. Promising antimicrobial and antioxidant extracts of murta leaves (Ugni molinae Turcz.): Shelf-life extension and food safety. Food Packaging and Shelf Life 1: 77-85.

Hoffmann, A. 2005. Flora silvestre de Chile zona araucana. $5^{\text {ta }}$ ed. Fundación Claudio Gay, Santiago, Chile. 258 pp.

Hossain, M., M. Sharma, J. Da Silva \& P. Pathak. 2010. Seed germination and tissue culture of Cymbidium giganteum Wall. ex Lindl. Scientia Horticulturae 123: 479-487.

ISTA. 2001. International rules for seed testing 2001. Seed Science and Technology 29(2): 28-29.

Je, J., H. Chen, C. Song \& CH. OH Lim. 2014. Arabidopsis DREB2C modulates ABA biosynthesis during germination. Biochemical and Biophysical Research Communications 452: 91-98.

Karssen, C., D. Brinkhorst-Van Der Swan, A. Breekland \& M. KoORNNEEF. 1983. Induction of dormancy during seed development by endogenous abscisic acid: studies on abscisic acid deficient genotypes of Arabidopsis thaliana (L.) Heynh. Planta 157: 158-165.

Karssen, C. \& E. LACKA. 1986. A revision of the hormone balance theory of seed dormancy: studies on gibberellin and/or abscisic acid-deficient mutants of Arabidopsis thaliana. In: M. Bopp (ed.), Plant growth substances, pp. 85-118. Springer-Verlag, Berlin.

Kermode, A.R. 2005. Role of abscisic acid in seed dormancy. Journal of Plant Growth Regulation 24: 319-344.

Khanna, P., A. Kumar, R. Chandra \& V. Verma. 2013. Germination behaviour of seeds of Withania somnifera (L.) Dunal: a high value medicinal plant. Physiology and Molecular Biology of Plants 19(3): 449-454.

Kim, Y., I. Ahn, A. Khan, M. Kamran, M. Waqas, J. Lee, D. KIm, S. JANG \& I. LeE. 2014. Regulation of endogenous gibberellins and abscisic acid levels during different seed collection periods in Panax ginseng. Horticulture, Environment, and Biotechnology 55: 166-174.

Kondhare, K.R., P. Hedden, P.S. Kettlewel, C. Farrell \& J.M. Monaghan. 2014. Use of the hormone-biosynthesis inhibitors fluridone and paclobutrazol to determine the effects of altered abscisic acid and gibberellin levels on pre-maturity a-amylase formation in wheat grains. Journal of Cereal Science 60: 210-216.

Koornneef, M., L. BentsinK \& H. Hilhorst. 2002. Seed dormancy and germination. Current Opinion in Plant Biology 5: 3336.

Kucera, B., M.A. Cohn \& G. Leubner-Metzger. 2005. Plant hormone interactions during seed dormancy release and germination. Seed Science Research 15: 281-307.

Kusumoto, D., S. Chae, K. Mukaida, K. Yoneyama, D. Joel \& Y. TAKEUCHI. 2006. Effects of fluridone and norflurazon 
on conditioning and germination of Striga asiatica seeds. Plant Growth Regulation 48: 73-78.

Larson, C., C. Gómez, M. Sánchez \& D. Ríos. 2006. Inducción de caulogénesis indirecta en Eucalyptus globulus. Bosque 27(3): 250-257.

Linkies, A. \& G. Leubner-Metzger. 2012. Beyond gibberellins and abscisic acid: how ethylene and jasmonates control seed germination. Plant Cell Reports 31: 253-270.

Lobo, M., Ó. Delgado, J. Régulo, E. Fernández \& C. Medina. 2007. Categorización de la germinación y la latencia en semillas de chirimoya (Annona cherimola L.) y guanábana (Annona muricata L.), como apoyo a programas de conservación de germoplasma. Agronomía Colombiana 25(2): 231-244.

Millar, A., J. Jacobsen, J. Ross, C. Helliwell, A. Poole, G. Scofield, J. Reid \& F. Gubler. 2006. Seed dormancy and ABA metabolism in Arabidopsis and barley: the role of ABA 8-hydroxylase. The Plant Journal 45: 942-954.

Moradi, K. \& M. Otroshy. 2012. A combination of chemical scarification and 6-benzylaminopurine (BAP) treatment promote seed germination in Dracocephalum kotschyi seeds. Trakia Journal of Sciences 10(3): 26-29.

Nonogaki, H., G. Bassel \& J. Bewley. 2010. Germination - still a mystery. Plant Science 179: 574-581.

Ogawa, M., A. Hanada, Y. Yamauchi, A. Kuwahara, Y. Kamiya \& S. Yamaguchi. 2003. Gibberellin biosynthesis and response during Arabidopsis seed germination. The Plant Cell 15: 1591-1604.

Pedroza-Manrique, J. \& W. Tupaz-Villacorte. 2008. Micropropagación de Ilex kunthiana Triana \& Plachon (Aquifoliaceae), una especie de gran importancia en programas de revegetalización. Revista Colombiana de Biotecnología 10(2): 72-84.

Pereira-Netto, A.B., M. Carvalho-Oliveira, J. Ramírez \& L. GalagovsKy. 2006. Shooting control in Eucalyptus grandis X E. urophylla hybrid: comparative effects of 28-homocastasterone and a 5a-monofluoro derivative. Plant Cell, Tissue and Organ Culture 86: 329-335.

Piotrowska, A. \& A. Bajguz. 2011. Conjugates of abscisic acid, brassinosteroids, ethylene, gibberellins, and jasmonates. Phytochemistry 72: 2097-2112.

Piskurewicz, U., V. Turecková, E. Lacombe \& L. Lopez-Molina. 2009. Far-red light inhibits germination through DELLAdependent stimulation of $\mathrm{ABA}$ synthesis and $\mathrm{ABI}_{3}$ activity. The EMBO Journal 28: 2259-2271.

Popova, L. 1995. Effect of fluridone on plant development and stress-induced ABA accumulation in Vicia faba L. Bulgarian Journal of Plant Physiology 21(2-3): 42-50.

Rayorath, P., W. Khan, R. Palanisamy, S. Mackinnon, R. Stefanova, S. Hankins, A. Critchley \& B. Prithiviraj.
2008. Extracts of the brown seaweed Ascophyllum nodosum induce gibberellic acid $\left(\mathrm{GA}_{3}\right)$-independent amylase activity in barley. Planth Growth Regulation 27: 370-379.

Rodriguez, M. 2011. La propagation d'écotypes sélectionnés de murtilla (Ugni molinae Turcz.), une baie endémique du Chili: étude de la germination et stratégies de multiplication in vitro. Tesis doctoral. Institut des Sciences et Industries du Vivant et de 1'Environnement, AgroParisTech, Ecole Doctorale ABIES, Paris, Francia. 232 pp.

Rodríguez, M., M. Chacón \& R. CARrillo. 2014a. Efecto de la concentración y de los componentes del medio de cultivo MS sobre la germinación in vitro de Ugni molinae. Bosque 35(1): 123-126.

Rodríguez, M., M. Latsague, M. Chacón \& P. Astorga. 2014b. Inducción in vitro de callogénesis y organogénesis indirecta a partir de explantes de cotiledón, hipocótilo y hoja en Ugni molinae. Bosque 35(1): 115-122.

Sawada, Y., T. Katsumata, J. Kitamura, H. Kawaide, M. Akajima, T. Sami, K. Nakaminami, T. Kurahashi, W. Mitsuhashi, Y. Inoue \& T. Toyomasu. 2008. Germination of photoblastic lettuce seeds is regulated via the control of endogenous physiologically active gibberellin content, rather than of gibberellin responsiveness. Journal of Experimental Botany 59: 3383-3393.

Smith-Ramírez, C., J. Armesto \& J. Figueroa. 1998. Flowering, fruiting and seed germination in Chilean rain forest myrtaceae: ecological and phylogenetic constraints. Plant Ecology 136: 119-131.

Song, S., T. QI, C. Wasternack \& D. XIE. 2014. Jasmonate signaling and crosstalk with gibberellin and ethylene. Current Opinion in Plant Biology 21: 112-119.

Steber, C. \& P. McCourt. 2001. A Role for brassinosteroids in germination in Arabidopsis. Plant Physiology 125: 763769.

Sun, K., Z. Zhang, B. GaO, Z. WAnG, D. Xu, J. Jin \& X. LiU. 2012. Adsorption of diuron, fluridone and norflurazon on singlewalled and multi-walled carbon nanotubes. Science of the Total Environment 439: 1-7.

Suwalsky, M. \& M. Avello. 2014. Antioxidant capacity of Ugni molinae fruit extract on human erythrocytes: an in vitro study. Journal of Membrane Biology 247: 703-712.

Wilson, R., H. Kim, A. BaKshi \& B. Binder. 2014a. The ethylene receptors ethylene response 1 and ethylene response have contrasting roles in seed germination of Arabidopsis during salt stress. Plant Physiology 165: 1353-1366.

Wilson, M., M. Basu, G. Bhaskara, P. Verslues \& E. Haswell. 2014b. Plastid osmotic stress activates cellular stress responses in Arabidopsis. Plant Physiology 165: 119-128.

Recibido: 06.01.15

Aceptado: 01.12.15 